\title{
The distribution of prime ideals of a Dedekind domain
}

\author{
Anne P. Grams
}

Let $G$ be an abelian group, and let $S$ be a subset of $G$. Necessary and sufficient conditions on $G$ and $S$ are given in order that there should exist a Dedekind domain $D$ with class group $G$ with the property that $S$ is the set of classes that contain maximal ideals of $D$. If $G$ is a torsion group, then $S$ is the set of classes containing the maximal ideals of $D$ if and only if $S$ generates $G$. These results are used to determine necessary and sufficient conditions on a family $\left\{H_{\lambda}\right\}$ of subgroups of $G$ in order that there should exist a Dedekind domain $D$ with class group $G$ such that $\left\{G / H_{\lambda}\right\}$ is the family of class groups of the set of overrings of $D$. Several applications are given.

\section{Introduction}

In [3], Claborn proved that each abelian group $G$ is the class group of a Dedekind domain $D$. Claborn extended his results in [4] and [5], Chapter III, to prove that some restrictions can be imposed on the prime ideal structure of $D$. Leedham-Green established similar results in [8]. The results of [4] enable Claborn to obtain a number of interesting examples - for instance, a Dedekind domain $D_{1}$, not a principal ideal domain, such that each proper overring of $D_{1}$ is a principal ideal domain,

Received 4 September 1974. Much of the research for this paper was done while the author was a student of Professor Robert Gilmer. The author Wrshes to acknowledge all the suggestions, comments, and help that Professor Gilmer gave her. 
and a Dedekind domain $D_{2}$ such that $D_{2}$ does not have the QR-property (cf. [7]), but each proper overring of $D_{2}$ has the $Q R$-property. In [9], Chapter 3, Levitz uses Claborn's results to give several other interesting examples.

In this paper, we investigate two questions concerning Dedekind domains and their class groups that are much in the same vein as those considered by $\mathrm{Claborn}$ and Leedham-Green. Specifically, we ask:

(1) Let $G$ be an abelian group, and let $S$ be a subset of $G$. Determine necessary and sufficient conditions on $G$ and $S$ in order that there should exist a Dedekind domain $D$ with class group isomorphic to $G$ under an isomorphism $\Phi$, with the property that $\Phi(S)$ is the set of elements of the class group of $D$ determined by a maximal ideal of $D .1$

(2) Let $G$ be an abelian group, and let $\left\{H_{\lambda}\right\}$ be a family of subgroups of $G$. Determine necessary and sufficient conditions on $G$ and $\left\{H_{\lambda}\right\}$ in order that there should exist a Dedekind domain $D$ with class group $G$ such that $\left\{G / H_{\lambda}\right\}$ is the family of class groups of the set of overrings of $D$.

Before proceeding further, some comment on (2) is appropriate. If $D$ is an integral domain with identity with quotient field $K$, then an overring of $D$ is a subring of $K$ that contains $D$. If $D$ is a Dedekind domain and if $J$ is an overring of $D$, then there is a canonical homomorphism of the class group $C(D)$ of $D$ onto the class group $C(J)$ of $J$ induced by the mapping $A+A J$ of the group of nonzero fractional ideals of $D$ onto the group of nonzero fractional ideals of $J$. The kernel $H_{J}$ of this homomorphism is generated by the set of classes $\{\bar{M} \mid M$ is a maximal ideal of $D$ and $M J=J\} \quad[2, \mathrm{p} .800]$. Thus $C(J) \simeq C(D) / H_{J}$. For convenience, we use the phrase "G/H is the class group of $J$ " to mean that the subgroup $B$ of $G$ is the kernel of the canonical homomorphism of $G=C(D)$ onto $C(J)$. It is in this sense that we intend question (2).

We answer questions (1) and (2) in $\$ 1$ and $\$ 2$. In $\S 3$ we give several applications of our results. One of the examples produced relates to the 1 This question was suggested to Professor Gilmer by Joe L. Mott. 
question of whether each nonzero element of a domain of finite character has at most a finite number of non-associate irreducible divisors (cf. [ $]$, p. 87]).

\section{Partitions of the class group}

Let $D$ be a Dedekind domain with class group $C(D)$. We partition $C(D)$ into two subsets $S$ and $T$, where $S$ consists of the classes that contain a maximal ideal of $D$ and $T$ consists of the classes that do not contain a maximal ideal. In our first proposition, two properties of $S$ are established that will play a key role in our answer to question (1).

PROPOSITION 1.1. The set $S$ has the following properties:

(1) $S$ generates $G$;

(2) if $s_{1}, \ldots, s_{k}$ are elements of $s$ and $n_{1}, \ldots, n_{k}$ are nonnegative integers, then there exist elements $r_{1}, \ldots, r_{t}$ of $S$ distinct from the $s_{i}$ and nonnegative integers $m_{1}, \ldots, m_{t}$ such that $\sum_{i=1}^{k} n_{i} s_{i}+\sum_{i=1}^{t} m_{i} r_{i}=0$.

Proof. (1) If $A$ is a nonzero fractional ideal of $D$, then there are maximal ideals $M_{1}, \ldots, M_{k}$ of $D$ and integers $n_{1}, \ldots, n_{k}$ such that $A=M_{1}^{n_{1}} \ldots M_{k}^{n_{k}}$. Hence $\bar{A}=n_{1} \bar{M}_{1}+\ldots+n_{k} \bar{M}_{k}$, and $S$ generates $C(D)$.

(2) This is a form of the approximation theorem for Dedekind domains. $/ /$

In order to provide a converse to Proposition 1.1 and an answer to question ( 1 ), we need a theorem of Claborn. For completeness, we give his terminology and theorem here.

Let $X$ be a set, and let $z^{(X)}$ denote the free abelian group based on $X$. We let $z_{+}^{(X)}$ be the set of elements $\sum n_{\lambda} x_{\lambda}$ in $z^{(X)}$ such that each $n_{\lambda}$ is nonnegative. If $X^{\prime}$ is a subset of $X$, then the restriction map $\rho: z^{(X)}+z^{\left(X^{\prime}\right)}$ defines a group homomorphism which is a surjection. 
If $P$ is a subset of $z_{+}^{(X)}$, we say that $P$ is finitely dense in $z^{(X)}$ if $\rho(P)=z_{+}^{\left(X^{\prime}\right)}$ for each finite subset $X^{\prime}$ of $X ; P$ is dense if $\rho(P)=Z_{+}^{\left(X^{\prime}\right)}$ for each subset $X^{\prime}$ of $X$ with $\operatorname{card}\left(X^{\prime}\right)<\operatorname{card}(X)$. (If $X$ is countable, then the conditions finitely dense and dense are equivalent; finitely dense is equivalent to Claborn's condition $(\alpha)$ in [4].)

THEOREM 1.2 (Claborn [5, p. 78]). Let $X$ be a set and $P$, contained in $Z_{+}^{(X)}$, a dense subset. Let $H$ be the subgroup of $Z^{(X)}$ generated by $P$. Then there exists a Dedekind domain $D$ with set $\left\{M_{x}\right\}_{x \in X}$ of maximal ideals such that the class group $C(D)$ is isomorphic to $Z^{(X)} / H$ under an isomorphism which sends $x+H$ to $\bar{M}_{x}$ for each $x \in X$.

We observe that if $H$ is a subgroup of $Z^{(X)}$, if $P=H \cap Z_{+}^{(X)}$, and if $P$ is finitely dense in $z^{(X)}$, then $P$ generates $H$. For if $h=n_{1} x_{1}+\ldots+n_{k} x_{k} \in H$, let $n_{i(1)}, \ldots, n_{i(s)}$ be the negative integers occurring among the integers $n_{1}, \ldots, n_{k}$. Then there exists an element $f \in P$ whose coefficient on $x_{i(j)}$ is $-n_{i(j)}, 1 \leq j \leq s$. Hence $h+p \in H \cap z_{+}^{(X)}=P$, so that $P$ generates $H$. This proves the following lemma.

LEMMA 1.3. Let $z^{(X)}$ be the free abelian group based on the set $X$. Let $H$ be a subgroup of $Z^{(X)}$, and let $P$ be the subset of $H$ consisting of the elements all of whose coefficients are nornegative. If $P$ is finitely dense, then $P$ generates $H$.

We now provide an answer to question (I).

THEOREM 1.4. Let $G$ be an abelian group, and let $S$ be a subset of $G$ satisfying the following two conditions:

(1) $S$ generates $G$; 
(2) if $\left\{s_{1}, \ldots, s_{k}\right\}$ is a finite subset of $S$, then there exist positive integers $n_{1}, \ldots, n_{k}$, elements $r_{1}, \ldots, r_{t}$ of $S$, and nonnegative integers $m_{1}, \ldots, m_{t}$ such that $n_{1} s_{1}+\ldots+n_{k} s_{k}+m_{1} r_{1}+\ldots+m_{t} r_{t}=0$.

Then there exists a Dedekind domain $D$ whose class group $C(D)$ is isomorphic to $G$ under an isomorphism $\Phi$ such that for each maximal ideal $M$ of $D, \Phi(\bar{M}) \in S$, and conversely, for each $s \in S$, there is a maximal ideal $M$ of $D$ such that $\Phi(\bar{M})=s$. Moreover, if $\lambda$ is any infinite cardinal greater than or equal to the cardinality of $G$, then $D$ can be chosen to have $\lambda$ maximal ideals and such that for each $s \in S$, there are $\lambda$ maximal ideals $M$ of $D$ such that $\Phi(\bar{M})=s$.

Proof. Let $\Lambda$ be an indexing set for $S$, and let $\Delta$ be an infinite set of cardinality greater than or equal to that of $G$. Let $T=\left\{T_{\lambda}\right\}_{\lambda \in \Lambda}$ be a partition of $\Delta$ into $|\Lambda|$ disjoint sets, each containing $|\Delta|$ elements. Let $J=\sum_{\delta \in \Delta} 2 x_{\delta}$ be the free abelian group based on the set $\left\{x_{\delta}\right\}_{\delta \in \Delta}$. Define a homomorphism $\psi: J \rightarrow G$ as follows:

$$
\psi\left(x_{\delta}\right)=s_{\lambda} \text { if } \delta \in T_{\lambda}
$$

and, in general,

$$
\psi\left(\sum m_{i} x_{i}\right)=\sum m_{i} \psi\left(x_{\delta_{i}}\right) .
$$

Since $S$ generates $G, \psi$ maps $J$ onto $G$. Let $H$ be the kernel of $\psi$, and let $P$ be the subset of $H$ consisting of the elements all of whose coefficients are nonnegative. We shall verify that $P$ is dense. It will then follow from Lema 1.3 that $P$ generates $H$. Hence by Theorem 1.2, there exists a Dedekind domain $D$ with maximal ideals $\left\{M_{\delta}\right\}_{\delta \in \Delta}$ and class group $C(D)$ such that $C(D)$ is isomorphic to $J / H$ under an isomorphism $\tau$ determined by the relations $\tau\left(x_{\delta}+H\right)=\bar{M}_{\delta}$. We claim that $D$ is the desired Dedekind domain. To see that this true, let $\bar{\psi}: J / H \rightarrow G$ be the induced isomorphism from $\psi$, and let $\Phi=\bar{\psi} \tau^{-1} ; \Phi$ is an isomorphism from $C(D)$ onto $G$. If $M_{\delta}$ is a maximal ideal of $D$, then 
$\Phi\left(\bar{M}_{\delta}\right)=\bar{\psi}\left(\tau^{-1}\left(\bar{M}_{\delta}\right)\right)=\bar{\psi}\left(x_{\delta}+H\right)=\psi\left(x_{\delta}\right) \in S$. Conversely, if $s_{\lambda} \in S$, pick $\delta \in T_{\lambda}$. Then $\Phi\left(\bar{M}_{\delta}\right)=\bar{\psi}\left(x_{\delta}+H\right)=\psi\left(x_{\delta}\right)={ }_{\lambda}{ }_{\lambda}$.

It remains to show that $P$ is dense. Let $X^{\prime}$ be a subset of $\left\{x_{\delta}\right\}$ such that $\left|X^{\prime}\right|<|\Delta|$, and let $f \in \sum_{x^{\prime} \in X^{\prime}} Z x^{\prime}$. Label the subscripts of the nonzero coefficients occurring in $f$ as $T_{f}=\left\{n_{1}, \ldots, n_{p}\right\}$. These integers (viewed as elements of $\Delta$ ) belong to at most $r$ elements of $T$ say $T_{\lambda_{1}}, \ldots, T_{\lambda_{v}}$. To simplify the notation, set $U_{i}=T_{\lambda_{i}} \cap T_{f} \cdot$ By hypothesis, there exist positive integers $k_{1}, \ldots, k_{v}$, elements $s_{\gamma_{1}}, \ldots, s_{\gamma_{t}}$ of $s$ and nonnegative integers $m_{1}, \ldots, m_{t}$ such that

$$
\sum_{i=1}^{v} k_{i} s \lambda_{i}+\sum_{i=1}^{t} m_{i} s \gamma_{i}=0
$$

By multiplying by a suitably large positive integer, we may assume that

$$
k_{i} \geq \sum_{n_{j} \in U_{i}} n_{j}
$$

Since $\left|X^{\prime}\right|<|\Delta|=\left|T_{\lambda}\right|$, we can select $d_{i} \in T_{\lambda_{i}}-X^{\prime}, 1 \leq i \leq v$. Similarly, for $1 \leq j \leq t$, we can select an element $c_{j} \in T_{\gamma_{j}}-\left(X^{\prime} \cup\left\{d_{1}, \ldots, d_{v}\right\}\right)$. Then if $g=f+\left(k_{1}-\sum_{n_{j} \in U_{1}} n_{j}\right) x_{d_{1}}+\ldots+\left(k_{v}-\sum_{n_{j} \in U_{v}} n_{j}\right) x_{d_{v}}+$

$$
+m_{1} x_{c_{1}}+\ldots+m_{t} x_{c_{t}}
$$

then $g$ projects onto $f$ under the restriction map induced by $X^{\prime}$. Moreover $g$ belongs to $P$ since all its coefficients are nonnegative and since 


$$
\begin{aligned}
\psi(g)=\left(\sum_{n_{j} \in U_{1}} n_{j}\right) s_{\lambda_{1}}+\ldots & +\left(\sum_{n_{j} \in U_{v}} n_{j}\right) s_{\lambda_{v}}+\left(k_{1}-\sum_{n_{j} \in U_{1}} n_{j}\right) s_{\lambda_{1}}+\ldots+ \\
& +\left(k_{v}-\sum_{n_{j} \in U_{v}} n_{j}\right) s_{\lambda_{v}}+m_{1} s_{\gamma_{1}}+\ldots+m_{t} \gamma_{t}=0 .
\end{aligned}
$$

Thus $P$ is dense, and this completes the proof of the theorem. //

It is an interesting consequence of Theorem 1.4 that i:f $S$ is a generating set for $G$ satisfying condition (2) of the theorem, then $S$ also satisfies the seemingly stronger condition (2) of Proposition 1.1.

We observe that if each element of $S$ has finite order or if $-S=\{-\boldsymbol{B} \mid s \in S\} \subseteq S$, then $S$ satisfies condition (2) of Theorem 1.4. Hence the following two corollaries follow easily from the theorem.

COROLLARY 1.5. Let $G$ be an abelian torsion group, and let $S$ be a subset of $G$. Then there exists a Dedekind domain with class group (isomorphic to) $G$ such that the classes which contain maximal ideals are precisely the elements of $S$ if and only if $S$ generates $G$.

COROLLARY 1.6. Let $G$ be an abelian group, let $S$ be a subset of $G$, and let $S^{\prime}=S \cup-S$. Then there exists a Dedekind domain with class group $G$ such that the classes that contain maximal ideals are precisely the elements of $S^{\prime}$ if and only if $S$ generates $G$.

\section{Class groups of overrings}

If $D$ is a Dedekind domain with class group $C(D)$ and if $J$ is an overring of $D$, then the class group of $J$ is isomorphic to a factor group of $C(D)$. It need not be true, however, that if $B$ is a subgroup of $C(D)$, then there is an overring of $D$ with class group $C(D) / H$. For example, Claborn [4, p. 253] and Levitz [9] construct Dedekind domains with cyclic class groups (both finite and infinite) such that all overrings are principal ideal domains, and hence have trivial class group. Question (2) is concerned with this type of problem. In order to provide an answer, we need the following result concerning the structure of the set of overrings of a Dedekind domain.

THEOREM 2.1 [6, p. 817]. Let $D$ be a Dedekind domain, and let $J$ be an overring of $D$. Then there exists a vnique collection $\left\{M_{\lambda}\right\}$ of 
maximal ideals of $D$ such that $J=\cap D_{M_{\lambda}}$. Precisely, $\left\{M_{\lambda}\right\}=\{M \mid M$ is a maximal ideal of $D$ and $M J \subset J\} .^{2}$

Let $D$ be a Dedekind domain with class group $G$, and let $\left\{G / H_{\lambda}\right\}$ be the family of factor groups of $G$ that are class groups of overrings of $D$. By our convention, $\left\{H_{\lambda}\right\}$ is the collection of kernels of the canonical map from $G$ onto $C(J)$, where $J$ ranges over the set of all overrings of $D$. Let $S$ be the subset of $G$ that consists of all classes containing a maximal ideal. With this notation, we have the following result. The proof is straightforward and will be omitted.

PROPOSITION 2.2. $\left\{H_{\lambda}\right\} \quad i_{8}$ the family of subgroups of $G$ generated. by the family of subsets of $S$.

The next result provides a converse to Proposition 2.2.

THEOREM 2.3. Let $G$ be an abelian group, and let $S$ be a subset of $G$ that generates $G$. Then there exists a Dedekind domain $D$ with class group $G$ such that the set of class groups of overrings of $D$ is $\left\{G / H_{\lambda}\right\}$, where $\left\{H_{\lambda}\right\}$ is the family of subgroups generated by the subsets of $S$.

Proof. By Corollary 1.6, there exists a Dedekind domain $D$ with class group $G$ such that

$$
S^{\prime}=S \cup-S=\{\bar{M} \mid M \text { is a maximal ideal of } D\} \text {. }
$$

We show that $D$ is the desired domain.

Let $B \in\left\{H_{\lambda}\right\}$. If $H=G$, let $M$ be a maximal ideal of $D$, and let $J=D_{M}$. Then $C(J)=\{0\}=G / H$. If $H \subset G$, let $J=\cap\left\{D_{M}|\bar{M}| H\right\}$. Then $P J=J$ if and only if $P \notin\{M|\bar{M}| H\}$ - that is, if and only if $P \in\{M \mid \bar{M} \in H\}$. Hence $C(J)=G / H$.

Conversely, let $J$ be an overring of $D$. The class group of $J$ is

2 Dedekind domains are not characterized by this property - that is, each overring is a unique intersection of localizations. In fact, if $D$ is a Prüfer domain, then distinct sets of maximal ideals of $D$ determine distinct overrings of $D$ if and only if there exists, for each maximal ideal $M$ of $D$, a finitely generated ideal $A$ contained in $M$ such that $M$ is the only maximal ideal of $D$ that contains $A[7, \mathrm{p} .283]$. 
$G / H$, where $H$ is generated by $\{\bar{M} \mid M J=J\}=H \cap S^{\prime}$. Since $S^{\prime}=S \cup-S, H$ is also generated by a subset of $S$. //

The following theorem answers question (2). It is a direct consequence of Theorem 2.3.

THEOREM 2.4. Let $G$ be an abelian group, let $H=\left\{H_{\lambda}\right\}$ be a collection of subgroups of $G$, and let $H^{\prime}=\left\{G / H_{\lambda}\right\}$ be the corresponding family of factor groups of $G$. Suppose that there exists a set $S$ of generators for $G$ such that $H$ is the family of subgroups generated by the subsets of $S$. Then there exists a Dedekind domain $D$ with class group $G$ such that $H^{\prime}$ is the family of class groups of the set of overrings of $D$.

\section{Examples}

EXAMPLE 3.1. A family $\left\{D_{\lambda}\right\}$ of domains with common quotient field $K$ is said to be of finite character if each element of $K$ is a unit in all but a finite number of the domains $D_{\lambda}$. Exercise 25 (a) in Bourbaki $[1, p .87]$ asserts that if $D$ is an intersection of a family $\left\{V_{\lambda}\right\}$ of valuation rings of finite character, then each nonzero element of $D$ has at most a finite number of nonassociate irreducible divisors. The hint given is to show that if $x \in D-\{0\}$ is a nonunit in precisely $n$ of the valuation rings and if $y_{1}, \ldots, y_{n+1}$ are $n+1$ nonassociate irreducible divisors of $x$, then $\left(y_{i}, y_{1} \ldots g_{i} \cdots y_{n+1}\right)_{v}=D$, where the caret signifies that $y_{i}$ is omitted from the product and where the ideal on the left is the $v$-ideal generated by $y_{i}$ and $y_{1} \ldots g_{i} \ldots y_{n+1}$. If the statement in the hint were true, then it would follow easily that $x$ has at most $n$ nonassociate irreducible divisors. We shall use the results of $\$ 2$ to give an example where this is not true. This will show that the "fact" given in the hint is false. Moreover, the example produced leads us to believe that the Bourbaki exercise is false, although we know of no counterexample.

For each positive integer $n>1$, let $c_{n}$ denote the cyclic group of order $n$, and let $G$ be the direct sum of the groups $\left\{c_{n}\right\}_{n>1}$. Let 
$S=\{(\overline{1}, 0, \ldots),(0, \overline{1}, 0, \ldots), \ldots\}$ be the standard generating set for $G$. (We use the same symbol $\bar{I}$ for the generator of each $c_{n} \cdot$ ) By Corollary 1.5, there exists a Dedekind domain $D$ with class group $G$ such that the classes which contain maximal ideals are precisely the elements of $S$. Moreover, we can assume that for each $s \in S$, there are infinitely many maximal ideals $M$ of $D$ such that $\bar{M}=\boldsymbol{s}$. We claim that $D$ has the following property.

Let $n$ and $m$ be positive integers with $1<n \leq m$. Then there is a nonzero element $x$ of $D$ such that $x$ is a nonumit in precisely $n$ valuation overrings of $D$, but $x$ has at least $m$ nonassociate irreducible divisors $y_{1}, \ldots, y_{m}$. Moreover, $\left(y_{1}, y_{2} \ldots y_{m}\right)_{v} \neq D$.

To see that this is true, let $\varepsilon_{m}=(0, \ldots, \overline{1}, 0, \ldots)$ be the element of $S$ with the $\bar{I}$ in the $m$-th slot. We can choose $n$ distinct maximal ideals $M_{1}, \ldots, M_{n}$ of $D$ such that $\bar{M}_{i}=\varepsilon_{m}, 1 \leq i \leq n$. Then the following ideals of $D$ are principal:

$$
\begin{gathered}
\left(y_{1}\right)=M_{1}^{m} \\
\left(y_{2}\right)=M_{1}^{m-1} M_{2} \\
\vdots \\
\left(y_{m-1}\right)=M_{1}^{2} M_{2}^{m-2} \\
\left(y_{m}\right)=M_{2}^{m-n+2} M_{3} \ldots M_{n} .
\end{gathered}
$$

Each $y_{i}$ is irreducible, for if any maximal ideals are omitted from the factorization of $\left(y_{i}\right)$, the resulting ideal is not principal. For example, $M_{1}^{m-2} M_{2}$ is not principal since

$$
\overline{M_{1}^{m-2} M_{2}}=(m-2) \varepsilon_{m}+\varepsilon_{m}=(m-1) \varepsilon_{m} \neq 0
$$

in $C(D)$.

Let $x=y_{1} \ldots y_{m}$. Then $x$ is a nonunit in precisely the valuation rings $D_{M_{1}}, \ldots, D_{M_{n}}$. Moreover, $x$ has at least $m$ nonassociate 
irreducible divisors $y_{1}, \ldots, y_{m}$, and $\left(y_{1}, y_{2} \ldots y_{m}\right)_{v} \subseteq\left(M_{1}\right)_{v} \neq D$.

EXAMPLE 3.2. If $D$ is an integral domain with identity, then $D$ is said to have the $Q R$-property if each overring of $D$ is a quotient ring of $D$. A Dedekind domain has the $Q R$-property if and only if its class group is a torsion group. This is an example of a Dedekind domain $D$ with mixed class group such that each proper overring of $D$ has the $Q R$-property; moreover, the torsion part of $C(D)$ is not of bounded order. Let $\left\{n_{i}\right\}_{i=1}^{\infty}$ be a strictly increasing sequence of positive integers with $n_{1}>1$. Let $G$ be the direct sum of the family $\{z\} \cup\left\{c_{n_{i}}\right\}$ of groups.

Set $s=(1,0,0, \ldots), s_{1}=(-1, \overline{1}, 0, \ldots)$, $s_{2}=(-1,0, \overline{1}, 0, \ldots)$, and, in general, $s_{i}=(-1,0, \ldots, \overline{1}, 0, \ldots)$. By Theorem 1.4, there exists a Dedekind domain $D$ with class group $G$ such that $S=\left\{s, s_{1}, s_{2}, \ldots\right\}$ is the set of classes determined by maximal ideals of $D$. It is easy to see that if $S^{\prime}$ is a nonempty subset of $S$ and if $B$ is the subgroup of $G$ generated by $S^{\prime}$, then $G / H$ is torsion. Hence each proper overring of $D$ has the $Q R$-property.

EXAMPLE 3.3. We construct a Dedekind domain such that the set of class groups of overrings is a prescribed set of cyclic groups. Let $S=\left\{n_{i}\right\}_{i \in I}$ be a set of integers with the property that $I \in S$ and that for each $n, m \in S$, their greatest common divisor also belongs to $S$. Then by Theorem 2.4, there exists a Dedekind domain $D$ with class group $Z$ such that $\{C(J) \mid J$ is an overring of $D\}=\left\{C_{n_{i}}\right\}_{i \in I} \cup\{(0), z\}$.

EXAMPLE 3.4. In [2], Claborn investigated conditions under which a Dedekind domain contains a semi-prime element - that is, an element that does not belong to the square of any maximal ideal. If $D$ is a Dedekind domain constructed as in Theorem 1.4, then $D$ contains semi-prime elements. In fact, each nonzero element of $D$ gives rise to a semi-prime element in the following way. Suppose that $d \in D-\{0\}$ and that (d) $=M_{1}^{n_{1}} \ldots M_{r}^{n^{p}}$ is the prime factorization of $(d)$. Then in $C(D)$, 
the relation $n_{1} \bar{M}_{1}+\ldots+n_{p} \bar{M}_{r}=0$ holds. For each maximal ideal $M$ of $D$, there are infinitely many maximal ideals $P_{i}$ of $D$ such that $\bar{M}=\bar{P}_{i}$. Hence for $1 \leq i \leq r$, we can select $n_{i}$ maximal ideals $P_{i(1)}, \ldots, P_{i\left(n_{i}\right)}$ of $D$ such that $\bar{M}_{i}=\bar{P}_{i(j)}, 1 \leq j \leq n_{i}$, where $P_{i(j)}=P_{k(8)}$ if and only if $i=k$ and $j=s$. Since

$$
\begin{gathered}
\sum_{j=1}^{n_{1}} \bar{P}_{1(j)}+\ldots+\sum_{j=1}^{n_{r}} \bar{P}_{r(j)}=0 \text {, the ideal } \\
P_{1(1)} \ldots P_{1\left(n_{1}\right)} \ldots P_{r(1)} \ldots P_{r\left(n_{r}\right)}=\left(d^{\prime}\right)
\end{gathered}
$$

is principal, and $d^{\prime}$ is a semi-prime element.

\section{References}

[1] N. Bourbaki, Éléments de mathématique. Algèbre commutative; Chapitre 7, Diviseurs (Actualités Scientifiques et Industrielles, 1314. Hermann, Paris, 1965).

[2] Luther Claborn, "Dedekind domains: overrings and semi-prime elements", Pacific J. Math. 15 (1965), 799-804.

[3] Luther Claborn, "Every abelian group is a class group", Pacific $J$. Math. 18 (1966), 219-222.

[4] Luther Claborn, "Specified relations in the ideal group", Michigan Math. J. 15 (1968), 249-255.

[5] Robert M. Fossum, The divisor class group of a Krulz domain (Ergebnisse der Mathematik und ihrer Grenzgebiete, 74. SpringerVerlag, Berlin, Heidelberg, New York, 1973).

[6] Robert W. Gilmer, Jr., "Integral domains which are almost Dedekind", Proc. Amer. Math. Soc. 15 (1964), 813-818.

[7] Robert W. Gilmer, Jr. and William J. Heinzer, "Overrings of Prüfer domains. II", J. Algebra 7 (1967), 281-302.

[8] C.R. Leedham-Green, "The class group of Dedekind domains", Trans. Amer. Math. Soc. 163 (1972), 493-500. 
[9] Kathleen Bulger Levitz, "Finiteness properties of certain rings", (Doctoral dissertation, Florida State University, Gainesville, 1971).

Department of Mathematics,

University of Tennessee at Nashville,

Nashville,

Tennessee,

USA. 